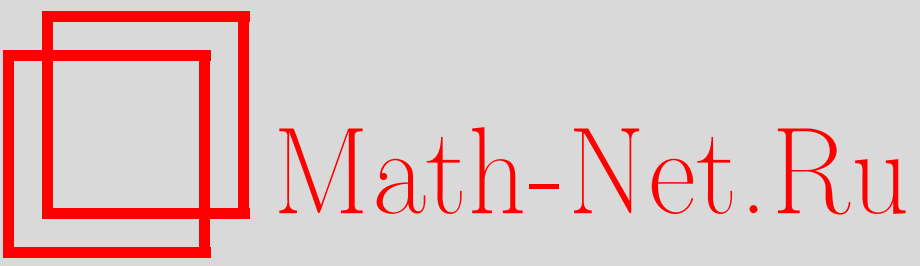

Д. Ф. Дигор, П. Ентел, М. Маринаро, В. А. Москаленко, Н. Б. Перкинс, О возможности образования связанных пар в периодической модели Андерсона, TMФ, 2001, том 127, номер 2, 304-316

DOI: https://doi.org/10.4213/tmf459

Использование Общероссийского математического портала Math-Net.Ru подразумевает, что вы прочитали и согласны с пользовательским соглашением

http://www. mathnet.ru/rus/agreement

Параметры загрузки:

IP: 54.198 .187 .58

26 апреля 2023 г., 05:37:48 
ТЕОРЕТИЧЕСКАЯ

И МАТЕМАТИЧЕСКАЯ

ФИЗИКА

Том 127, № 2

май, 2001

(C) 2001 г.

Д. Ф. Дигор ${ }^{*}$, П. Ентел ${ }^{\dagger}$, М. Маринаро ${ }^{\ddagger}$,

В. А. Москаленко*, Н.Б. Перкинс ${ }^{\S}$

\title{
О ВОЗМОЖНОСТИ ОБРАЗОВАНИЯ СВЯЗАННЫХ ПАР В ПЕРИОДИЧЕСКОЙ МОДЕЛИ АНДЕРСОНА
}

\begin{abstract}
Исследуется обобщенная периодическая модель Андерсона, описывающая две группы сильно коррелированных $(d$ и $f)$ электронов с локальной гибридизацией состояний и перескоком $d$-электронов между узлами решетки, с точки зрения возможности появления в ней связанных электронных пар. Атомный предел этой модели допускает точное решение на основе метода канонического преобразования. Ренормированный энергетический спектр локальной модели делится на низкоэнергетическую и высокоэнергетическую части, разделенные интервалом порядка энергии кулоновского отталкивания электронов. Проецирование гамильтониана на состояния низкоэнергетической части спектра приводит к появлению членов парного взаимодействия электронов, принадлежаших $d$ - и $f$-орбиталям, и к возможности их туннелирования между этими орбиталями. При этом слагаемые гамильтониана, обусловленные ионными энергиями и перескоками, оказываются сильно коррелированными и реализуются только между состояниями, которые не заполнены дважды. Полученный гамильтониан более не содержит сильных взаимодействий, которые оказываются подавленными квантовыми флуктуациями гибридизации состояний. После его линеаризации в приближении среднего поля установлен энергетический спектр квазичастиц и намечен способ самосогласования параметров порядка сверхпроводящей фазы. Для простоты все расчеты сделаны для симметричной периодической модели Андерсона, в которой энергии дважды заполненных $d$ - и $f$-орбиталей предполагаются одинаковыми.
\end{abstract}

\section{1. ВВЕДЕНИЕ}

Исследуемая нами периодическая модель Андерсона (ПМА) [1] состоит из двух сильно коррелированных подсистем $d$ - и $f$-электронов. Предполагается локальная гибридизация орбиталей и туннелирование $d$-электронов между узлами кристаллической решетки. Гамильтониан этой модели дополнен членами отталкивания $d$ - и $f$-электронов

\footnotetext{
* Институт прикладной физики Молдавской АН, Кишинев, Молдова. E-mail: statphys@asm.md

${ }^{\dagger}$ Duisburg University, Germany. E-mail: entel@thp.uni-duisburg.de

${ }^{\ddagger}$ Salerno University, Italy. E-mail: marinaro@sa.infn.it

$\S$ Объединенный институт ядерных исследований, Дубна, Московская область, Россия. E-mail: perkins@thsun1.jinr.ru
} 
(так называемое слагаемое Фаликова-Кимбелла) и имеет вид [2]

$$
\begin{aligned}
H & =H^{0}+H^{\prime}, \\
H^{0} & =H_{d}^{0}+H_{f}^{0}+H_{\mathrm{hyb}}+H_{\mathrm{FK}},
\end{aligned}
$$

где

$$
\begin{aligned}
H_{d}^{0} & =\varepsilon_{d} \sum_{i \sigma} d_{i \sigma}^{\dagger} d_{i \sigma}+U_{d} \sum_{i} n_{i \uparrow}^{d} n_{i \downarrow}^{d}, \\
H_{f}^{0} & =\varepsilon_{f} \sum_{i \sigma} f_{i \sigma}^{\dagger} f_{i \sigma}+U_{f} \sum_{i} n_{i \uparrow}^{f} n_{i \downarrow}^{f}, \\
H_{\mathrm{hyb}} & =V \sum_{i \sigma}\left(f_{i \sigma}^{\dagger} d_{i \sigma}+d_{i \sigma}^{\dagger} f_{i \sigma}\right), \\
H_{\mathrm{FK}} & =G \sum_{i} n_{i}^{d} n_{i}^{f}, \\
H^{\prime} & =\sum_{i j \sigma} t(j-i) d_{j \sigma}^{\dagger} d_{i \sigma} .
\end{aligned}
$$

Здесь $d_{i \sigma}^{\dagger}\left(d_{i \sigma}\right)$ и $f_{i \sigma}^{\dagger}\left(f_{i \sigma}\right)$ - операторы рождения (уничтожения) $d$ - и $f$-электронов, соответственно; $n_{i \sigma}^{d}=d_{i \sigma}^{\dagger} d_{i \sigma}$ и $n_{i \sigma}^{f}=f_{i \sigma}^{\dagger} f_{i \sigma}$ - операторы числа частиц в узле $i$ со спином $\sigma ; U_{d}$ и $U_{f}$ - энергии одноузельного отталкивания; $\varepsilon_{d}=\bar{\varepsilon}_{d}-\mu, \varepsilon_{f}=\bar{\varepsilon}_{f}-\mu$ являются ионными энергиями $d$ - и $f$-орбиталей, отсчитанными от химического потенциала $\mu ; V$ - матричный элемент одноузельной гибридизации состояний, который для простоты считается постоянным. Благодаря гибридизации (2в) и перескокам (2д) обе группы локализованных электронов становятся подвижными и могут участвовать в проводимости.

Свойства локального гамильтониана (1б) изучались многими авторами [3]-[8]. В работах [9]-[13] и в приведенных там ссылках можно найти обзор результатов теоретических и экспериментальных исследований сильно коррелированных электронных систем. Ниже мы следуем в основном определениям, содержащимся в работе [2]. Обозначим через $\phi_{n}\left(E_{\lambda}\right)$ собственные функции и через $E_{\lambda}$ собственные значения матрицы локального гамильтониана (1б). При этом индекс $n=1, \ldots, 16$ нумерует исходные ионные квантовые состояния в одном узле с различным числом частиц и полным спином. Квантовое число $\lambda$ относится к ренормированным состояниям, полученным после диагонализации гамильтониана атомного предела. Эти состояния также являются одноузельными, но в отличие от состояний, описываемых индексом $n$, учитывают гибридизацию. Функции $\phi_{n}\left(E_{\lambda}\right)$ определяют структуру $S$-матрищы, диагонализуюшей локальньй гамильтониан, $S_{n \lambda}=\phi_{n}\left(E_{\lambda}\right)$. $S$-матрица определяет также закон преобразования трансфер-операторов Хаббарда [14] $X^{m n}=|m\rangle\langle n|$ исходного представления к диагональному представлению $Y^{\lambda \lambda^{\prime}}=\left|E_{\lambda}\right\rangle\left\langle E_{\lambda^{\prime}}\right|$ согласно формулам

$$
\begin{aligned}
X^{m n} & =\sum_{\lambda \lambda^{\prime}} \phi_{m}\left(E_{\lambda}\right) \phi_{n}^{*}\left(E_{\lambda^{\prime}}\right) Y^{\lambda \lambda^{\prime}}, \\
Y^{\lambda \lambda^{\prime}} & =\sum_{m n} \phi_{m}^{*}\left(E_{\lambda}\right) \phi_{n}\left(E_{\lambda^{\prime}}\right) X^{m n} .
\end{aligned}
$$

5 Теоретическая и математическая физика, т. 127, № 2, 2001 г. 
Эти формулы определяют также преобразование операторов рождения и уничтожения $d$ - и $f$-электронов. Так, например, оператор $d_{\uparrow}$, до преобразования имевший вид

$$
\begin{aligned}
d_{\uparrow}= & X^{1,2}+X^{4,6}+\frac{1}{\sqrt{2}}\left(X^{5,7}+X^{5,10}\right)+\frac{1}{\sqrt{2}}\left(X^{10,12}-X^{7,12}\right)+ \\
& +X^{11,14}+X^{3,9}+X^{8,13}+X^{15,16}
\end{aligned}
$$

после преобразования переходит в

$$
\begin{aligned}
S^{-1} d_{\uparrow} S= & \sum_{\lambda=2,3} \phi_{2}\left(E_{\lambda}\right) Y^{1, \lambda}+\sum_{\lambda=2,3} \phi_{3}\left(E_{\lambda}\right) Y^{\lambda, 9}+\frac{1}{\sqrt{2}} \sum_{\lambda=4,5} \phi_{5}\left(E_{\lambda}\right) Y^{\lambda, 10}+ \\
& +\sum_{a=6,7,8} \sum_{\lambda=4,5}\left[\frac{1}{\sqrt{2}} \phi_{5}\left(E_{\lambda}\right) \phi_{7}\left(E_{a}\right)+\phi_{4}\left(E_{\lambda}\right) \phi_{6}\left(E_{a}\right)\right] Y^{\lambda, a}+ \\
& +\sum_{a=6,7,8} \sum_{\lambda=12,13}\left[-\frac{1}{\sqrt{2}} \phi_{7}\left(E_{a}\right) \phi_{12}\left(E_{\lambda}\right)+\phi_{8}\left(E_{a}\right) \phi_{13}\left(E_{\lambda}\right)\right] Y^{a, \lambda}+ \\
& +\frac{1}{\sqrt{2}} \sum_{\lambda=12,13} \phi_{12}\left(E_{\lambda}\right) Y^{10, \lambda}+\sum_{\lambda=14,15}\left(\phi_{14}\left(E_{\lambda}\right) Y^{11, \lambda}+\phi_{15}\left(E_{\lambda}\right) Y^{\lambda, 16}\right)
\end{aligned}
$$

С помощью (5) и аналогичных формул для других операторов рождения и уничтожения легко определить среднее число $d$ - и $f$-электронов в локальном приближении, их одночастичные функции Грина и плотности электронных состояний.

\section{2. НИЗКОЭНЕРГЕТИЧЕСКИЙ ГАМИЛЬТОНИАН}

Рассмотрим проекцию гамильтониана ПМА на подпространство низкоэнергетических состояний. Это позволит получить эффективный гамильтониан, которьй содержит конкурирующие магнитные и сверхпроводящие флуктуации. Используем каноническое преобразование полного гамильтониана

$$
\widetilde{H}=S^{-1} H S=H_{\mathrm{r}}^{0}+H_{\mathrm{r}}^{\prime}
$$

где

$$
\begin{aligned}
H_{\mathrm{r}}^{0} & =\sum_{i \lambda} E_{\lambda} Y_{i}^{\lambda \lambda}, \\
H_{\mathrm{r}}^{\prime} & =\sum_{i j \sigma} t(i-j) S^{-1} d_{i \sigma}^{\dagger} d_{j \sigma} S .
\end{aligned}
$$

Новые операторы $S^{-1} d_{\sigma} S\left(S^{-1} d_{\sigma}^{\dagger} S\right)$ определены равенствами вида (5) (более подробно см. [2]). Как и в [2], мы для простоты рассматриваем симметричную ПМА, которая соответствует случаю, когда ионные энергии дважды занятых $d$ - и $f$-состояний равны друг другу, т.е.

$$
2 \varepsilon_{d}+U_{d}=2 \varepsilon_{f}+U_{f}
$$


Это ограничение не имеет решающего значения, однако оно упрощает проблему и позволяет найти решения для собственных функций $\phi_{n}\left(E_{\lambda}\right)$ и соответствуюших собственных значений локального гамильтониана. Для самого низкого синглетного состояния $(\lambda=6)$ гибридизированной $(d-f)$-пары электронов имеем

$$
\begin{aligned}
E_{6} & =\varepsilon_{d}+\varepsilon_{f}+\frac{1}{2}(U+G)-\frac{1}{2} \sqrt{(U-G)^{2}+(4 V)^{2}}, \\
\phi_{6}\left(E_{6}\right) & =\frac{1}{2}\left[1-\frac{U-G}{\sqrt{(U-G)^{2}+(4 V)^{2}}}\right]^{\frac{1}{2}}, \\
\phi_{7}\left(E_{6}\right) & =-\frac{1}{\sqrt{2}} \operatorname{sign} V\left[1+\frac{U-G}{\sqrt{(U-G)^{2}+(4 V)^{2}}}\right]^{\frac{1}{2}},
\end{aligned}
$$

где $U$ - среднее значение двух энергий отталкиваюшего взаимодействия $U_{d}$ и $U_{f}$. При умеренной гибридизации $V \simeq 0.5$ эВ, большом $U \simeq 5$ эВ и $G \simeq 1$ эВ энергия синглетного основного состояния $\left|E_{6}\right\rangle$,

$$
E_{6} \simeq \varepsilon_{d}+\varepsilon_{f}+G-\frac{4 V^{2}}{U-G}
$$

содержит выигрыш энергии порядка энергии кондо-связи

$$
\frac{4 V^{2}}{U-G} \simeq \frac{2 V^{2}}{\varepsilon_{d}-\varepsilon_{f}}
$$

которая может оказать существенное влияние на процесс образования электронных пар в ПМА. Эта идея не нова, ранее она обсуждалась Хиршем [15]. Имеется и другая особенность данной модели, связанная с возможностью туннелирования электронных пар меж ду различными орбиталями. Идея о сушественной роли туннелирования электронных пар как целого впервые была сформулирована в связи с теорией сверхпроводимости систем с перекрываюшимися энергетическими зонами [16], [17] и относилась к переходам пар между разными зонами. Как будет показано ниже, низкоэнергетическая часть гамильтониана содержит парные взаимодействия, которые после учета перескоков $d$-электронов могут инициировать движение связанных электронных пар по кристаллической решетке.

Для доказательства этого утверждения воспользуемся проекционным методом [13]. Мы делим энергетический спектр локализованной системы на две части, разделенные интервалом порядка энергии отталкивания $U$. Собственные значения энергии $E_{\lambda}$ с квантовыми числами $\lambda=1,2, \ldots, 6,9,10,11$ (см. [2]) принадлежат к низкоэнергетической части спектра, тогда как те, что имеют значения $\lambda=7,8,12, \ldots, 16,-$ к высокоэнергетической части. Проекционный оператор имеет вид

$$
P=\Pi_{i} P_{i}, \quad P_{i}=\sum_{\lambda \in \alpha} Y_{i}^{\lambda \lambda}, \quad \alpha=1,2, \ldots, 6,9,10,11 .
$$

Ортогональный ему оператор -

$$
Q=1-P
$$


Благодаря условию полноты

$$
\sum_{\lambda=1}^{16} Y_{i}^{\lambda \lambda}=1
$$

ортогональный оператор содержит суммирования лишь по высокоэнергетическим состояниям.

Вместо единственного уравнения Шредингера для всего гильбертова пространства $H|\psi\rangle=E|\psi\rangle$ после проецирования возникают два уравнения Шредингера для двух подпространств. Для интересующего нас низкоэнергетического подпространства с волновой функцией $\left|\psi_{P}\right\rangle=P|\psi\rangle$ имеем следующее уравнение:

$$
\left(\widetilde{H}_{P P}-E\right)\left|\psi_{P}\right\rangle=0,
$$

где

$$
\widetilde{H}_{P P}=H_{P P}^{0}+H_{P P}^{\prime}-H_{P Q}^{\prime}\left(H_{Q Q}^{0}+H_{Q Q}^{\prime}-E\right)^{-1} H_{Q P}^{\prime}
$$

и

$$
\begin{aligned}
& H_{P P}^{0}=P H_{\mathrm{r}}^{0} P=\sum_{i} \sum_{\lambda \in \alpha} E_{\lambda} Y_{i}^{\lambda \lambda}, \\
& H_{P P}^{\prime}=P H_{\mathrm{r}}^{\prime} P .
\end{aligned}
$$

Последнее слагаемое в (16) описывает вклад от виртуальных квантовых переходов через исключенные высокоэнергетические уровни и имеет порядок величины $t^{2} / U$, где $t$ интеграл перескока между соседними узлами. Так как для энергетических параметров теории предполагается выполнение условий

$$
t \ll V \ll U, \quad \frac{t^{2}}{U} \ll \frac{V^{2}}{U-G},
$$

то последний член в (16) можно опустить и сохранить в качестве эффективного низкоэнергетического гамильтониана ПМА выражение

$$
\widetilde{H}_{P P}=H_{P P}^{0}+H_{P P}^{\prime} .
$$

В качестве примера применения проекционной техники рассмотрим оператор

$$
\begin{aligned}
\widetilde{d}_{\uparrow} \equiv & P S^{-1} d_{\uparrow} S P=\sum_{\lambda=2,3} \phi_{2}\left(E_{\lambda}\right) Y^{1, \lambda}+\sum_{\lambda=2,3} \phi_{3}\left(E_{\lambda}\right) Y^{\lambda, 9}+\frac{1}{\sqrt{2}} \phi_{5}\left(E_{\lambda}\right) Y^{\lambda, 10}+ \\
& +\sum_{\lambda=4,5}\left[\frac{1}{\sqrt{2}} \phi_{5}\left(E_{\lambda}\right) \phi_{7}\left(E_{6}\right)+\phi_{4}\left(E_{\lambda}\right) \phi_{6}\left(E_{6}\right)\right] Y^{\lambda, 6}
\end{aligned}
$$

который сушественно отличается от первоначальной формы (5). Исходное ионное представление гамильтониана через операторы рождения и уничтожения электронов оказывается более пригодным для анализа и интерпретации структуры возникшего проекционного гамильтониана. Поэтому над операторами (19) в дальнейшем выполняется обратное преобразование

$$
\widetilde{H}=S \widetilde{H}_{P P} S^{-1}=\widetilde{H}_{P P}^{0}+\widetilde{H}_{P P}^{\prime}
$$


Приведем примеры такого преобразования:

$$
\begin{aligned}
m_{\sigma}= & d_{\sigma}^{\dagger} d_{\sigma}, \quad n_{\sigma}=f_{\sigma}^{\dagger} f_{\sigma}, \\
\tilde{\tilde{d}}_{\sigma}= & d_{\sigma}\left(1-m_{\bar{\sigma}}\right)\left(1-n_{\sigma} n_{\bar{\sigma}}\right)+\frac{1}{4}\left[1-\frac{U-G}{\sqrt{(U-G)^{2}+(4 V)^{2}}}\right] \times \\
& \times\left[d_{\sigma}\left(1-n_{\sigma}\right)\left(m_{\bar{\sigma}}-n_{\bar{\sigma}}\right)+d_{\bar{\sigma}}^{\dagger} f_{\bar{\sigma}} f_{\sigma}\left(1-m_{\sigma}\right)-f_{\bar{\sigma}}^{\dagger} d_{\bar{\sigma}} f_{\sigma}\left(1-m_{\sigma}\right)\right]- \\
& -\frac{V}{\sqrt{(U-G)^{2}+(4 V)^{2}}} \times \\
& \times\left[f_{\bar{\sigma}}^{\dagger} d_{\bar{\sigma}} d_{\sigma}\left(1-n_{\sigma}\right)+d_{\bar{\sigma}}^{\dagger} f_{\bar{\sigma}} d_{\sigma}\left(1-n_{\sigma}\right)+f_{\sigma}\left(1-m_{\sigma}\right)\left(m_{\bar{\sigma}}-n_{\bar{\sigma}}\right)^{2}\right] \\
\tilde{\tilde{f}}_{\sigma}= & f_{\sigma}\left(1-n_{\bar{\sigma}}\right)\left(1-m_{\sigma} m_{\bar{\sigma}}\right)+ \\
& +\frac{1}{4}\left[1-\frac{U-G}{\sqrt{(U-G)^{2}+(4 V)^{2}}}\right] \times \\
& \times\left[f_{\sigma}\left(1-m_{\sigma}\right)\left(n_{\bar{\sigma}}-m_{\bar{\sigma}}\right)+f_{\bar{\sigma}}^{\dagger} d_{\bar{\sigma}} d_{\sigma}\left(1-n_{\sigma}\right)-d_{\bar{\sigma}}^{\dagger} f_{\bar{\sigma}} d_{\sigma}\left(1-n_{\sigma}\right)\right]- \\
& -\frac{V}{\sqrt{(U-G)^{2}+(4 V)^{2}}} \times \\
& \times\left[d_{\bar{\sigma}}^{\dagger} f_{\bar{\sigma}} f_{\sigma}\left(1-m_{\sigma}\right)+f_{\bar{\sigma}}^{\dagger} d_{\bar{\sigma}} f_{\sigma}\left(1-m_{\sigma}\right)+d_{\sigma}\left(1-n_{\sigma}\right)\left(n_{\bar{\sigma}}-m_{\bar{\sigma}}\right)^{2}\right] .
\end{aligned}
$$

В формулах (22а), (22б) для простоты опущены индексы узлов решетки. Полученные выражения показывают ограничения заполнения состояний, при которых уничтожение электрона данной орбитали с данным спином на данном узле возможно при условии, что нет электрона с противоположным спином данной орбитали. Эти ограничения хорошо известны для $(t-J)$-модели, которая может быть получена проекционной техникой из модели Хаббарда [13]. Наличие двух видов электронов в ПАМ делает ограничения заполнения более сложными и заменяет линейные формы операторов на нелинейные структуры (формулы (22а), (22б)). На основании этих формул для первого слагаемого в правой части (21) получаем

$$
\begin{aligned}
\widetilde{H}_{P P}^{0}= & \sum_{\lambda=1, \ldots, 5,6,9,10,11} E_{\lambda} Y^{\lambda \lambda}= \\
= & \varepsilon_{d} \sum_{\sigma} m_{\sigma}\left(1-m_{\bar{\sigma}}\right)\left(1-n_{\sigma}\right)\left(1-n_{\bar{\sigma}}\right)+\varepsilon_{f} \sum_{\sigma} n_{\sigma}\left(1-n_{\bar{\sigma}}\right)\left(1-m_{\sigma}\right)\left(1-m_{\bar{\sigma}}\right)+ \\
& +V \sum_{\sigma}\left(f_{\sigma}^{\dagger} d_{\sigma}+d_{\sigma}^{\dagger} f_{\sigma}\right)\left[\left(1-m_{\bar{\sigma}}\right)\left(1-n_{\bar{\sigma}}\right)-\frac{E_{6}\left(m_{\bar{\sigma}}-n_{\bar{\sigma}}\right)^{2}}{\sqrt{(U-G)^{2}+(4 V)^{2}}}\right]+ \\
& +\frac{1}{2} E_{6} \sum_{\sigma} m_{\sigma}\left(1-n_{\bar{\sigma}}\right) n_{\bar{\sigma}}\left(1-n_{\sigma}\right)+\frac{1}{2}\left[E_{6}-E_{9}-2 E_{6} \phi_{6}^{2}\left(E_{6}\right)\right] \sum_{\sigma} d_{\sigma}^{\dagger} f_{\bar{\sigma}}^{\dagger} d_{\bar{\sigma}} f_{\sigma}+ \\
& +E_{6} \phi_{6}^{2}\left(E_{6}\right)\left(m_{\uparrow}-n_{\uparrow}\right)\left(m_{\downarrow}-n_{\downarrow}\right)+\frac{1}{2} E_{6} \phi_{6}^{2}\left(E_{6}\right) \sum_{\sigma}\left(d_{\sigma}^{\dagger} d_{\bar{\sigma}}^{\dagger} f_{\bar{\sigma}} f_{\sigma}+f_{\sigma}^{\dagger} f_{\bar{\sigma}}^{\dagger} d_{\bar{\sigma}} d_{\sigma}\right)+ \\
& +\frac{1}{2} E_{9} \sum_{\sigma}\left[2 m_{\sigma} n_{\sigma}\left(1-m_{\bar{\sigma}}\right)\left(1-n_{\bar{\sigma}}\right)+m_{\sigma} n_{\bar{\sigma}}\left(1-m_{\bar{\sigma}}\right)\left(1-n_{\sigma}\right)\right] .
\end{aligned}
$$


Второе слагаемое, обусловленное перескоками, принимает вид

$$
\widetilde{H}_{P P}^{\prime}=\sum_{i j \sigma} t(i-j) \tilde{\tilde{d}}_{i \sigma}^{\dagger} \tilde{\tilde{d}}_{j \sigma}
$$

Так как оператор (24) пропорционален параметру $t$, то возможно опустить в нем те вклады, которые имеют порядок малости $t V /(U-G)$. В результате имеем более простое выражение

$$
\widetilde{H}_{P P}^{\prime}=\sum_{i j \sigma} t(i-j) d_{i \sigma}^{\dagger} d_{j \sigma}\left(1-m_{i \bar{\sigma}}\right)\left(1-m_{j \bar{\sigma}}\right)\left(1-n_{i \sigma} n_{i \bar{\sigma}}\right)\left(1-n_{j \sigma} n_{j \bar{\sigma}}\right)
$$

Следует обратить внимание на особенности низкоэнергетического гамильтониана (формулы $(23),(25))$. Одна из них состоит в том, что в этих формулах фигурируют многочастичные операторы, возникшие вследствие понижения энергии из-за исключения двойного заполнения узельных орбиталей. Так, например, первые два слагаемых (23) являются вкладами свободных ионных орбиталей с указанным ограничением на заполнение состояний. Третий член является вкладом, обусловленным гибридизацией состояний при наличии указанных ограничений. Флуктуации, обусловленные гибридизацией состояний, приводят к возникновению парных взаимодействий электронов с противоположными спинами, принадлежаших как к одной и той же, так и к различным орбиталям. Как видно из правой части (23), парные взаимодействия, пропорциональные $E_{6} \phi^{2}\left(E_{6}\right)$, отвечают притяжению в том случае, когда синглетное состояние $\left|E_{6}\right\rangle$ имеет отрицательную энергию $E_{6}$, что соответствует эффекту Кондо. Весовой множитель $\phi^{2}\left(E_{6}\right)$ определяет вероятность образования такой электронной пары на $d$ - и $f$-орбиталях. Из определения (9б) видно, что эта вероятность тем меншше, чем больше кулоновское отталкивание $U$ одноорбитальных электронов. Наличие межорбитального отталкивания $G$ способствует образованию этих пар в той мере, в которой оно уменьшает величину $U$. Подобная положительная роль межорбитального отталкивания отмечена в работе [18].

Другой важной особенностью полученного гамильтониана является наличие туннелирования синглетных состояний пар как целого между различными орбиталями. Выше была отмечена аналогия между этим туннелированием и межзонными переходами. Таким образом, полученньй эффективньй гамильтониан обнаруживает уже в локальном (нулевом) приближении парные взаимодействия, способствуюшие образованию синглетных электронных пар.

Делокализация электронов, определяемая вкладом перескоков (25), задается сильно нелинейным оператором. Эта нелинейность целиком обязана ограничениям занятости и может сама служить источником парных флуктуаций сверхпроводящего вида. Так как обе части эффективного гамильтониана (23) и (25) содержат только слабые локальные взаимодействия и слабый коррелированный перескок электронов, мы полагаем возможным в качестве первого шага использовать для дальнейшего исследования полученного гамильтониана метод среднего поля. 


\section{3. МЕТОД СРЕДНЕГО ПОЛЯ}

Запишем эффективньй низкоэнергетический гамильтониан, определенный слагаемым (23) (после его суммирования по узлам решетки) и вкладом (25), в виде

$$
\begin{aligned}
\widetilde{H}= & \varepsilon_{d} \sum_{i \sigma} d_{i \sigma}^{\dagger} d_{i \sigma}\left(1-m_{i \bar{\sigma}}\right)\left(1-n_{i \sigma} n_{\bar{\sigma}}\right)+\varepsilon_{f} \sum_{i \sigma} f_{i \sigma}^{\dagger} f_{i \sigma}\left(1-n_{i \bar{\sigma}}\right)\left(1-m_{i \sigma} m_{i \bar{\sigma}}\right)+ \\
& +V \sum_{i \sigma}\left(f_{i \sigma}^{\dagger} d_{i \sigma}+d_{i \sigma}^{\dagger} f_{i \sigma}\right)\left[\left(1-m_{i \bar{\sigma}}\right)\left(1-n_{i \bar{\sigma}}\right)-\frac{E_{6}\left(m_{i \bar{\sigma}}-n_{i \bar{\sigma}}\right)^{2}}{\sqrt{(U-G)^{2}+(4 V)^{2}}}\right]- \\
& -\left(2 \alpha_{1} V+\alpha_{2} E_{9}\right) \sum_{i \sigma}\left[d_{i \sigma}^{\dagger} f_{i \bar{\sigma}} d_{i \bar{\sigma}} f_{i \sigma}+m_{i \sigma} n_{i \bar{\sigma}}\left(1-m_{i \bar{\sigma}}\right)\left(1-n_{i \sigma}\right)\right]+ \\
& +E_{6} \alpha_{2} \sum_{i \sigma}\left[d_{i \sigma}^{\dagger} d_{i \bar{\sigma}}^{\dagger} f_{i \bar{\sigma}}^{\dagger} f_{i \sigma}+f_{i \sigma}^{\dagger} f_{i \bar{\sigma}}^{\dagger} d_{i \bar{\sigma}}^{\dagger} d_{i \sigma}+\right. \\
& \left.+\frac{1}{2} m_{i \sigma} m_{i \bar{\sigma}}\left(1-n_{i \sigma}\right)\left(1-n_{i \bar{\sigma}}\right)+\frac{1}{2} n_{i \sigma} n_{i \bar{\sigma}}\left(1-m_{i \sigma}\right)\left(1-m_{i \bar{\sigma}}\right)\right]+ \\
& +G \sum_{i}\left(m_{i \uparrow}-m_{i \downarrow}\right)^{2}\left(n_{i \uparrow}-n_{i \downarrow}\right)^{2}+ \\
& +\sum_{i j \sigma} t(i-j) d_{i \sigma}^{\dagger} d_{j \sigma}\left(1-m_{i \bar{\sigma}}\right)\left(1-m_{j \bar{\sigma}}\right)\left(1-n_{\sigma} n_{i \bar{\sigma}}\right)\left(1-n_{\sigma} n_{j \bar{\sigma}}\right) .
\end{aligned}
$$

Это выражение следует привести к квадратичному виду с целью получения гамильтониана $H_{\mathrm{MF}}$ свободных квазичастиц. Одновременно необходимо указать способ вычисления соответствующих средних и квазисредних величин. Так как нас интересует возможность реализации сверхпроводящего состояния, то в преобразованном к квадратичному виду гамильтониане необходимо сохранить операторы синглетных $d-d, f-f$ и $d-f$ электронных пар. При этом появятся величины

$$
\bar{m}_{\sigma}=\left\langle d_{\sigma}^{\dagger} d_{\sigma}\right\rangle, \quad \bar{n}_{\sigma}=\left\langle f_{\sigma}^{\dagger} f_{\sigma}\right\rangle, \quad \bar{p}_{\sigma}=\left\langle d_{\sigma}^{\dagger} f_{\sigma}\right\rangle,
$$

равные средним числам электронов на узле, и квазисредние Боголюбова [19] вида

$$
\gamma_{\sigma}^{d}=\left\langle d_{\bar{\sigma}} d_{\sigma}\right\rangle, \quad \gamma_{\sigma}^{f}=\left\langle f_{\bar{\sigma}} f_{\sigma}\right\rangle, \quad \gamma_{\sigma}^{m}=\left\langle d_{\bar{\sigma}} f_{\sigma}\right\rangle=\left\langle f_{\bar{\sigma}} d_{\sigma}\right\rangle .
$$

Перечисленные средние значения (27), (28) определены самосогласованно на основе равенства

$$
\langle A\rangle=\frac{\operatorname{Tr}\left(A e^{-\beta H_{\mathrm{MF}}}\right)}{\operatorname{Tr} e^{-\beta H_{\mathrm{MF}}}} .
$$

Квадратичный гамильтониан имеет вид

$$
\begin{aligned}
& H_{\mathrm{MF}}=E_{0}+ \\
& \quad+\sum_{\mathbf{k} \sigma}\left[E_{d}(\mathbf{k}, \sigma) d_{\mathbf{k} \sigma}^{\dagger} d_{\mathbf{k} \sigma}+E_{f}(\mathbf{k}, \sigma) f_{\mathbf{k} \sigma}^{\dagger} f_{\mathbf{k} \sigma}+E_{m}(\mathbf{k}, \sigma) d_{\mathbf{k} \sigma}^{\dagger} f_{\mathbf{k} \sigma}+E_{m}^{*}(\mathbf{k}, \sigma) f_{\mathbf{k} \sigma}^{\dagger} d_{\mathbf{k} \sigma}\right]+ \\
& \quad+\sum_{\mathbf{k}}\left[\Delta_{d}(\mathbf{k}) d_{\mathbf{k} \uparrow}^{\dagger} d_{-\mathbf{k} \downarrow}^{\dagger}+\Delta_{d}^{*}(\mathbf{k}) d_{-\mathbf{k} \downarrow} d_{\mathbf{k} \uparrow}+\Delta_{f}(\mathbf{k}) f_{\mathbf{k} \uparrow}^{\dagger} f_{-\mathbf{k} \downarrow}^{\dagger}+\Delta_{f}^{*}(\mathbf{k}) f_{-\mathbf{k} \downarrow} f_{\mathbf{k} \uparrow}\right]+ \\
& \quad+\sum_{\mathbf{k}}\left[\Delta_{m}(\mathbf{k}) d_{\mathbf{k} \uparrow}^{\dagger} f_{-\mathbf{k} \downarrow}^{\dagger}+\bar{\Delta}_{m}(\mathbf{k}) f_{\mathbf{k} \uparrow}^{\dagger} d_{-\mathbf{k} \downarrow}^{\dagger}+\Delta_{m}^{*}(\mathbf{k}) f_{-\mathbf{k} \downarrow} d_{\mathbf{k} \uparrow}+\bar{\Delta}_{m}^{*}(\mathbf{k}) d_{-\mathbf{k} \downarrow} f_{\mathbf{k} \uparrow}\right] .(30)
\end{aligned}
$$


Здесь нами использовано квазидискретное импульсное представление для электронных операторов и интеграла перескока,

$$
\begin{aligned}
d_{i \sigma} & =\frac{1}{\sqrt{N}} \sum_{\mathbf{k}} d_{\mathbf{k} \sigma} e^{-i \mathbf{k} \mathbf{R}_{i}}, \\
f_{i \sigma} & =\frac{1}{\sqrt{N}} \sum_{\mathbf{k}} f_{\mathbf{k} \sigma} e^{-i \mathbf{k} \mathbf{R}_{i}}, \\
t(i-j) & =\frac{1}{N} \sum_{\mathbf{k}} \varepsilon(\mathbf{k}) e^{-i \mathbf{k}\left(\mathbf{R}_{i}-\mathbf{R}_{j}\right)},
\end{aligned}
$$

где $\varepsilon(\mathbf{k})$ - энергия свободных электронов. Коэффициенты квадратичной формы (30) состоят из двух слагаемых. Первое слагаемое отвечает локальному гамильтониану и не зависит от энергии зонных электронов. Второе слагаемое определяется перескоками электронов и пропорционально энергии зонных электронов. Отметим, что все средние, содержащие операторы, принадлежащие разным узлам, пропорциональны этой зонной энергии, так как в отсутствие перескоков они равнялись бы нулю. Так как энергия перескоков считается малой величиной, то в дальнейшем в сумме слагаемых, содержаших одноузельные и межузельные средние, последними для простоты будем пренебрегать. После этих упрощений коэффициенты квадратичной формы (30) принимают вид

$$
\begin{aligned}
E_{d}(\mathbf{k}, \sigma)= & \varepsilon_{d \sigma}+\varepsilon(\mathbf{k})\left[\left\langle\left(1-m_{\bar{\sigma}}\right)\left(1-n_{\sigma} n_{\bar{\sigma}}\right)\right\rangle\right]^{2}- \\
& -\varepsilon(\mathbf{k})\left\langle d_{\sigma}^{\dagger} d_{\bar{\sigma}}^{\dagger}\left(1-n_{\sigma} n_{\bar{\sigma}}\right)\right\rangle\left\langle d_{\bar{\sigma}} d_{\sigma}\left(1-n_{\sigma} n_{\bar{\sigma}}\right)\right\rangle, \\
E_{f}(\mathbf{k}, \sigma)= & \varepsilon_{f \sigma}+\varepsilon(\mathbf{k})\left\langle f_{\sigma}^{\dagger} d_{\sigma}\left(1-m_{\bar{\sigma}}\right) n_{\bar{\sigma}}\right\rangle\left\langle d_{\sigma}^{\dagger} f_{\sigma}\left(1-m_{\bar{\sigma}}\right) n_{\bar{\sigma}}\right\rangle- \\
& -\varepsilon(\mathbf{k})\left\langle f_{\sigma}^{\dagger} d_{\bar{\sigma}}^{\dagger}\left(1-m_{\sigma} n_{\bar{\sigma}}\right)\right\rangle\left\langle d_{\bar{\sigma}} f_{\sigma}\left(1-m_{\sigma} n_{\bar{\sigma}}\right)\right\rangle, \\
E_{m}(\mathbf{k}, \sigma)= & \varepsilon_{m \sigma}+\varepsilon(\mathbf{k})\left\langle f_{\sigma}^{\dagger} d_{\sigma}\left(1-m_{\bar{\sigma}}\right) n_{\bar{\sigma}}\right\rangle\left\langle\left(1-m_{\bar{\sigma}}\right)\left(1-n_{\sigma} n_{\bar{\sigma}}\right)\right\rangle- \\
& -\varepsilon(\mathbf{k})\left\langle f_{\sigma}^{\dagger} d_{\bar{\sigma}}^{\dagger}\left(1-m_{\sigma}\right) n_{\bar{\sigma}}\right\rangle\left\langle d_{\bar{\sigma}} d_{\sigma}\left(1-m_{\sigma} n_{\bar{\sigma}}\right)\right\rangle, \\
\Delta_{d}(\mathbf{k}, \sigma)= & \delta_{d \sigma}-\varepsilon(\mathbf{k})\left\langle d_{\bar{\sigma}} d_{\sigma}\left(1-n_{\sigma} n_{\bar{\sigma}}\right)\right\rangle\left\langle\left(1-m_{\bar{\sigma}}\right)\left(1-n_{\sigma} n_{\bar{\sigma}}\right)\right\rangle, \\
\Delta_{f}(\mathbf{k}, \sigma)= & \delta_{f \sigma}-\varepsilon(\mathbf{k})\left\langle f_{\bar{\sigma}} d_{\sigma}\left(1-m_{\bar{\sigma}}\right) n_{\sigma}\right\rangle\left\langle d_{\sigma}^{\dagger} f_{\sigma}\left(1-m_{\bar{\sigma}}\right) n_{\bar{\sigma}}\right\rangle, \\
\Delta_{m}(\mathbf{k}, \sigma)= & \delta_{m \sigma}-\varepsilon(\mathbf{k})\left\langle d_{\bar{\sigma}} d_{\sigma}\left(1-n_{\sigma} n_{\bar{\sigma}}\right)\right\rangle\left\langle d_{\bar{\sigma}}^{\dagger} f_{\bar{\sigma}}\left(1-m_{\sigma}\right) n_{\sigma}\right\rangle- \\
& -\varepsilon(\mathbf{k})\left\langle f_{\bar{\sigma}} d_{\sigma}\left(1-m_{\bar{\sigma}}\right) n_{\sigma}\right\rangle\left\langle\left(1-m_{\bar{\sigma}}\right)\left(1-n_{\sigma} n_{\bar{\sigma}}\right)\right\rangle,
\end{aligned}
$$

где одноузельные слагаемые (индекс узла опущен)

$$
\begin{aligned}
\varepsilon_{d \sigma} & \equiv \varepsilon_{d}\left\langle\left(1-2 m_{\bar{\sigma}}\right)\left(1-n_{\sigma} n_{\bar{\sigma}}\right)\right\rangle-\varepsilon_{f}\left\langle m_{\bar{\sigma}}\left(n_{\sigma}-n_{\bar{\sigma}}\right)^{2}\right\rangle- \\
& -V\left[\left\langle\left(d_{\bar{\sigma}}^{\dagger} f_{\bar{\sigma}}+f_{\bar{\sigma}}^{\dagger} d_{\bar{\sigma}}\right)\left(1-n_{\sigma}\right)\right\rangle+\frac{E_{6}\left\langle\left(d_{\bar{\sigma}}^{\dagger} f_{\bar{\sigma}}+f_{\bar{\sigma}}^{\dagger} d_{\bar{\sigma}}\right)\left(1-2 n_{\sigma}\right)\right\rangle}{\sqrt{(U-G)^{2}+(4 V)^{2}}}\right]+ \\
& +E_{6} \phi_{6}^{2}\left(E_{6}\right)\left\langle m_{\bar{\sigma}}-n_{\bar{\sigma}}\right\rangle+\frac{1}{2}\left(E_{6}-E_{9}\right)\left\langle n_{\bar{\sigma}}\left(1-m_{\bar{\sigma}}\right)\left(1-n_{\sigma}\right)-m_{\bar{\sigma}} n_{\sigma}\left(1-n_{\bar{\sigma}}\right)\right\rangle,
\end{aligned}
$$


a $\varepsilon_{f \sigma}$ получается из $\varepsilon_{d \sigma}$ взаимной заменой величин $d$ и $f$,

$$
\begin{aligned}
\varepsilon_{m \sigma}=\varepsilon_{d} & \left\langle f_{\sigma}^{\dagger} d_{\sigma} n_{\bar{\sigma}}\left(1-2 m_{\bar{\sigma}}\right)\right\rangle+\varepsilon_{f}\left\langle f_{\sigma}^{\dagger} d_{\sigma} m_{\bar{\sigma}}\left(1-2 n_{\bar{\sigma}}\right)\right\rangle- \\
& -V\left\langle f_{\sigma}^{\dagger} d_{\bar{\sigma}}^{\dagger} f_{\bar{\sigma}} d_{\sigma}+f_{\sigma}^{\dagger} f_{\bar{\sigma}}^{\dagger} d_{\bar{\sigma}} d_{\sigma}\right\rangle\left[1+\frac{2 E_{6}}{\sqrt{(U-G)^{2}+(4 V)^{2}}}\right]+ \\
& +\frac{1}{2}\left(E_{6}-E_{9}\right)\left\langle f_{\sigma}^{\dagger} d_{\sigma}\left(m_{\bar{\sigma}}-n_{\bar{\sigma}}\right)^{2}\right\rangle+E_{6} \phi_{6}^{2}\left(E_{6}\right)\left\langle d_{\bar{\sigma}}^{\dagger} f_{\bar{\sigma}}\right\rangle+ \\
& +\left[\frac{1}{2}\left(E_{6}-E_{9}\right)-E_{6} \phi_{6}^{2}\left(E_{6}\right)\right]\left\langle f_{\bar{\sigma}}^{\dagger} d_{\bar{\sigma}}\right\rangle
\end{aligned}
$$

Локальный вклад в сверхпроводящий параметр порядка $d$-электронов имеет вид

$$
\begin{aligned}
\delta_{d \sigma}= & -\varepsilon_{d}\left\langle d_{\bar{\sigma}} d_{\sigma}\left(1-n_{\sigma} n_{\bar{\sigma}}\right)\right\rangle-\varepsilon_{f}\left\langle d_{\bar{\sigma}} d_{\sigma} n_{\sigma}\left(1-n_{\bar{\sigma}}\right)\right\rangle-G\left\langle d_{\bar{\sigma}} d_{\sigma}\left(n_{\sigma}-n_{\bar{\sigma}}\right)^{2}\right\rangle- \\
& -V\left[\left\langle d_{\bar{\sigma}} f_{\sigma}\left(1-n_{\sigma}\right)\right\rangle+\frac{E_{6}\left\langle d_{\bar{\sigma}} f_{\sigma}\left(1-2 n_{\bar{\sigma}}\right)\right\rangle}{\sqrt{(U-G)^{2}+(4 V)^{2}}}\right]- \\
& -\frac{1}{2}\left(E_{6}-E_{9}\right)\left\langle d_{\bar{\sigma}} d_{\sigma} n_{\bar{\sigma}}\left(1-n_{\sigma}\right)\right\rangle+\frac{1}{2} E_{6} \phi_{6}^{2}\left(E_{6}\right)\left\langle d_{\bar{\sigma}} d_{\sigma}+f_{\bar{\sigma}} f_{\sigma}\right\rangle .
\end{aligned}
$$

Соответствуюшее выражение для $\delta_{f \sigma}$ получается из вышеприведенного заменой величин $d$ и $f$. Локальный вклад смешанного параметра порядка определяется выражением

$$
\begin{aligned}
\delta_{m \sigma}= & -\varepsilon_{d}\left\langle f_{\bar{\sigma}} d_{\sigma} n_{\sigma}\left(1-2 m_{\bar{\sigma}}\right\rangle-\varepsilon_{f}\left\langle f_{\bar{\sigma}} d_{\sigma} m_{\bar{\sigma}}\left(1-2 n_{\sigma}\right)\right\rangle+E_{6} \phi_{6}^{2}\left(E_{6}\right)\left\langle f_{\bar{\sigma}} d_{\sigma}-d_{\bar{\sigma}} f_{\sigma}\right\rangle-\right. \\
& -V\left[\left\langle f_{\bar{\sigma}} f_{\sigma}\left(1-m_{\bar{\sigma}}\right)+d_{\bar{\sigma}} d_{\sigma}\left(1-n_{\sigma}\right)\right\rangle+\frac{E_{6}\left\langle d_{\bar{\sigma}} d_{\sigma}\left(1-2 n_{\sigma}\right)+f_{\bar{\sigma}} f_{\sigma}\left(1-2 m_{\bar{\sigma}}\right)\right\rangle}{\sqrt{(U-G)^{2}+(4 V)^{2}}}\right]+ \\
& +\frac{1}{2}\left(E_{6}-E_{9}\right)\left\langle f_{\bar{\sigma}} d_{\sigma}\left[\left(1-m_{\bar{\sigma}}\right)\left(1-n_{\sigma}\right)+m_{\bar{\sigma}} n_{\sigma}\right]+d_{\bar{\sigma}} f_{\sigma}\right\rangle .
\end{aligned}
$$

Мы полагаем, что явление синглетной сверхпроводимости может реализоваться на основании парамагнитной фазы, что позволяет считать средние числа частиц и одночастичные энергии не зависящими от спинов, т.е.

$$
E_{\alpha}(\mathbf{k}, \sigma)=E_{\alpha}(\mathbf{k}, \uparrow)=E_{\alpha}(-\mathbf{k}, \downarrow), \quad \alpha=d, f, m
$$

Три параметра порядка $\Delta_{d}(\mathbf{k}), \Delta_{f}(\mathbf{k})$ и $\Delta_{m}(\mathbf{k})$ определены равенствами

$$
\begin{aligned}
\Delta_{\alpha}(\mathbf{k}) & =\Delta_{\alpha}(\mathbf{k}, \uparrow)-\Delta_{\alpha}(-\mathbf{k}, \downarrow), \quad \alpha=d, f, \\
\Delta_{m}(\mathbf{k}) & =\Delta_{m}(\mathbf{k}, \uparrow)=\bar{\Delta}_{m}(-\mathbf{k}, \downarrow) .
\end{aligned}
$$

После сделанных упрощений легко диагонализовать квадратичный гамильтониан (30). Для этого введем гамильтонову матрицу

$$
\widehat{H}_{\mathrm{MF}}^{0}=\left(\begin{array}{cccc}
E_{d}(\mathbf{k}) & E_{m}(\mathbf{k}) & \Delta_{d}(\mathbf{k}) & \Delta_{m}(\mathbf{k}) \\
E_{m}(\mathbf{k}) & E_{f}(\mathbf{k}) & \Delta_{m}(\mathbf{k}) & \Delta_{f}(\mathbf{k}) \\
\Delta_{d}^{*}(\mathbf{k}) & \Delta_{m}^{*}(\mathbf{k}) & -E_{d}(-\mathbf{k}) & -E_{m}(-\mathbf{k}) \\
\Delta_{m}^{*}(\mathbf{k}) & \Delta_{m}^{*}(\mathbf{k}) & -E_{m}(-\mathbf{k}) & -E_{f}(-\mathbf{k})
\end{array}\right)
$$


и биспиноры

$$
\hat{\psi}(\mathbf{k})=\left(\begin{array}{c}
d_{\mathbf{k} \uparrow} \\
f_{\mathbf{k} \uparrow} \\
d_{-\mathbf{k} \downarrow}^{\dagger} \\
f_{-\mathbf{k} \downarrow}^{\dagger}
\end{array}\right), \quad \hat{\psi}^{\dagger}(\mathbf{k})=\left(d_{\mathbf{k} \uparrow}^{\dagger} f_{\mathbf{k} \uparrow}^{\dagger} d_{-\mathbf{k} \downarrow} f_{-\mathbf{k} \downarrow}\right),
$$

которые позволяют переписать гамильтониан среднего поля (30) в виде

$$
H_{\mathrm{MF}}=E_{0}+\sum_{\mathbf{k}} \hat{\psi}^{\dagger}(\mathbf{k}) \widehat{H}_{\mathrm{MF}}^{0} \hat{\psi}(\mathbf{k})+\sum_{\mathbf{k}}\left(E_{d}(\mathbf{k})+E_{f}(\mathbf{k})\right)
$$

Для диагонализации матрищы (44) используем ортонормированную систему собственных функций $\chi_{n}\left(E_{\lambda}\right) \quad(n=1, \ldots, 4, \lambda=1, \ldots, 4)$ и собственных значений $E_{\lambda}$, удовлетворяющих уравнению

и условиям ортонормировки

$$
\widehat{H}_{\mathrm{MF}}^{0}\left(\begin{array}{c}
\chi_{1}\left(E_{\lambda}\right) \\
\chi_{2}\left(E_{\lambda}\right) \\
\chi_{3}\left(E_{\lambda}\right) \\
\chi_{4}\left(E_{\lambda}\right)
\end{array}\right)=E_{\lambda}\left(\begin{array}{c}
\chi_{1}\left(E_{\lambda}\right) \\
\chi_{2}\left(E_{\lambda}\right) \\
\chi_{3}\left(E_{\lambda}\right) \\
\chi_{4}\left(E_{\lambda}\right)
\end{array}\right)
$$

$$
\begin{aligned}
\sum_{m} \chi_{m}^{*}\left(E_{\lambda}\right) \chi_{m}\left(E_{\lambda^{\prime}}\right) & =\delta_{\lambda \lambda^{\prime}} \\
\sum_{\lambda} \chi_{m}^{*}\left(E_{\lambda}\right) \chi_{n}\left(E_{\lambda}\right) & =\delta_{m n}
\end{aligned}
$$

Соответствуюшие преобразования Боголюбова, которые диагонализуют $\widehat{H}_{\mathrm{MF}}^{0}$, имеют вид

$$
\begin{aligned}
d_{\mathbf{k} \uparrow} & =\sum_{\lambda} \chi_{1}\left(E_{\lambda}\right) \alpha_{\lambda}(\mathbf{k}), \\
f_{\mathbf{k} \uparrow} & =\sum_{\lambda} \chi_{2}\left(E_{\lambda}\right) \alpha_{\lambda}(\mathbf{k}), \\
d_{-\mathbf{k} \downarrow}^{\dagger} & =\sum_{\lambda} \chi_{3}\left(E_{\lambda}\right) \alpha_{\lambda}(\mathbf{k}), \\
f_{-\mathbf{k} \downarrow}^{\dagger} & =\sum_{\lambda} \chi_{4}\left(E_{\lambda}\right) \alpha_{\lambda}(\mathbf{k}),
\end{aligned}
$$

где "новые" операторы $\alpha_{\lambda}(\lambda=1, \ldots, 4)$ определены равенством

$$
\alpha_{\lambda}(\mathbf{k})=\chi_{1}^{*}\left(E_{\lambda}\right) d_{\mathbf{k} \uparrow}+\chi_{2}^{*}\left(E_{\lambda}\right) f_{\mathbf{k} \uparrow}+\chi_{3}^{*}\left(E_{\lambda}\right) d_{-\mathbf{k} \downarrow}^{\dagger}+\chi_{4}^{*}\left(E_{\lambda}\right) f_{-\mathbf{k} \downarrow}^{\dagger} \cdot
$$

Благодаря условиям (48) перестановочные свойства операторов $\alpha_{\lambda}$ сохраняются фермиевскими. Собственные значения энергии $E_{\lambda}^{\mathrm{S}}$ определяются из условия равенства нулю детерминанта $\left\|H_{n m}^{0}-E^{\mathrm{s}} \delta_{n m}\right\|$, что приводит к решениям

$$
\begin{aligned}
& E_{ \pm}^{\mathrm{s}} 2=\frac{1}{2}\left\{E_{d}^{2}+E_{f}^{2}+2 E_{m}^{2}+\left|\Delta_{d}\right|^{2}+\left|\Delta_{f}\right|^{2}+2\left|\Delta_{m}\right|^{2} \pm\right. \\
& \pm\left[\left(E_{d}^{2}+\left|\Delta_{d}\right|^{2}-E_{f}^{2}-\left|\Delta_{f}\right|^{2}\right)^{2}+4\left|E_{m}\left(E_{d}+E_{f}\right)+\Delta_{m}^{*} \Delta_{f}+\Delta_{m} \Delta_{f}^{*}\right|^{2}+\right. \\
& \left.\left.+4\left|\left(E_{d}-E_{f}\right) \Delta_{m}^{*}+E_{m}\left(\Delta_{f}^{*}-\Delta_{d}^{*}\right)\right|^{2}\right]^{\frac{1}{2}}\right\}
\end{aligned}
$$


Четыре собственных значения гамильтоновой матрицы (44) суть

$$
\begin{array}{ll}
E_{1}^{\mathrm{S}}(\mathbf{k})=-\sqrt{\left(E_{+}^{\mathrm{s}}\right)^{2}}, & E_{2}^{\mathrm{S}}(\mathbf{k})=-\sqrt{\left(E_{-}^{\mathrm{s}}\right)^{2}}, \\
E_{3}^{\mathrm{S}}(\mathbf{k})=+\sqrt{\left(E_{-}^{\mathrm{s}}\right)^{2}}, & E_{4}^{\mathrm{S}}(\mathbf{k})=+\sqrt{\left(E_{+}^{\mathrm{s}}\right)^{2}} .
\end{array}
$$

Нетрудно убедиться, что оба значения (51) положительны. Тем самым два собственных значения (52) отрицательны и два положительны. Спектр энергий квазичастиц оказывается хорошо определенным. Он зависит как от трех одночастичных энергий нормальной фазы системы, так и от трех параметров порядка сверхпроводяшей фазы. Полученные результаты следует дополнить условиями самосогласования параметров порядка сверхпроводящей фазы, а также определением химического потенциала системы. Эти условия фактически сводятся к трем нелинейным уравнениям для средних одноузельных чисел частиц $\bar{m}, \bar{n}$ и $\bar{p}$ и одного дополнительного условия для химического потенциала, а также к трем нелинейным уравнениям для локальных параметров порядка $\gamma_{d}$,

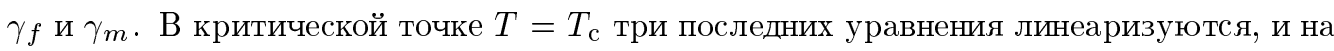
их основе возможно определение $T_{\mathrm{c}}$. Эта программа дальнейших исследований может ответить на вопрос, достаточны ли перечисленные выше эффекты для возникновения сверхпроводимости в рассматриваемой ПМА.

\section{4. ОБСУЖДЕНИЕ РЕЗУЛЬТАТОВ}

В работе получен эффективный гамильтониан сильно коррелированных $d$ - и $f$-электронов. Он содержит низкоэнергетические спиновые флуктуации, приводящие к коррелированному перескоку электронов между узлами решетки и к локальным парным взаимодействиям электронов с противоположными спинами, принадлежашими как к одинаковым, так и к разным орбиталям. Вместо ионных энергий $d$ - и $f$-электронов и кинетической энергии $d$-электронов, содержащихся в исходном гамильтониане ПМА, возникают сильно коррелированные величины, содержащие ограничения двойной занятости узлов решетки. Наличие двух электронных орбиталей приводит к двойному ограничению и к возникновению более сложных, чем в $(t-J)$-модели, нелинейных слагаемых гамильтониана.

Как следует из полученных результатов, имеется несколько факторов, способствующих образованию связанных электронных пар. Во-первых, отметим существование синглетного состояния с отрицательной энергией $E_{6}$. Это состояние содержит выигрыш энергии благодаря эффекту Кондо, приводящему к притяжению электронных пар. Во-вторых, сушествуют коррелированные переходы, которые порождают флуктуируюшие пары электронов с противоположными спинами, принадлежашие к обеим орбиталям. Третьим фактором является туннелирование электронных пар между различными орбиталями. Это явление в результате виртуальных переходов способно привести к дополнительному притяжению пар, подобно тому как это имеет место в теории многозонных сверхпроводников. Четвертым фактором является наличие кулоновского отталкивания одноузельных электронов, принадлежащих к разным орбиталям. Это отталкивание увеличивает вероятность образования электронных пар одной и той же орбитали 
в той мере, в какой межорбитальное отталкивание $G$ уменьшает значение кулоновского отталкивания одноорбитальных электронов $U$. Линеаризация полученного эффективного гамильтониана на основе приближения среднего поля привела к концепшии хорошо определенных квазичастиц.

Дальнейшее исследование полученных основных уравнений может ответить на вопрос, есть ли на самом деле сверхпроводимость в ПМА.

Благодарности. Мы (Д.Ф.Д. и В.А.М.) благодарим Высший совет по науке и технологическому развитию Правительства Республики Молдова за финансовую поддержку при выполнении данной работы. Один из авторов (В.А.М) благодарен университетам Салерно и Дуйсбурга за гостеприимство и финансовую поддержку. Мы (В.А.М и Н.Б.П) благодарны профессору Н. М. Плакиде и участникам его семинара за полезное обсуждение. Двое из нас (П.Е. и В.А.М.) благодарят комитет проекта Гейзенберг-Ландау за предоставленный грант и ЛТФ ОИЯИ за гостеприимство.

\section{Список литературы}

[1] P. W. Anderson. Phys. Rev. 1961. V. 124. P. 41.

[2] V. A. Moskalenko, P. Entel, M. Marinaro, N. B. Perkins, C. Holtfort. Hopping perturbation treatment of the Periodic Anderson Model around the atomic limit. Phys. Rev. B (in press); В. А. Москаленко, Н. Б. Перкинс. ТМФ. 1999. Т. 121. № 3. С. 464.

[3] M. W. Long. Z. Phys. B. 1988. V. 69. P. 409, 427; 1988. V. 71. P. 23.

[4] F. Mancini, M. Marinaro, Y. Nakano. Physica B. 1989. V. 159. P. 330.

[5] F. Mancini, M. Marinaro, Y. Nakano, C. Noce, A. Romano. Nuovo Cimento D. 1989. V. 11. P. 1709.

[6] C. Noce, A. Romano. J. Phys.: Condens. Matter. 1989. V. 1. P. 8347.

[7] C. Noce, A. Romano. Physica B. 1990. V. 160. P. 304.

[8] B. R. Alascio, R. Allub, A. Aligia. J. Phys. C. 1980. V. 13. P. 2869.

[9] G. Czycholl. Phys. Rep. 1986. V. 143. P. 277.

[10] P. Schlottmann. Phys. Rep. 1989. V. 181. P. 1.

[11] С. Г. Оөчинников. УФН. 1997. Т. 167. С. 1043.

[12] A. C. Hewson. The Kondo Problem to Heavy Fermions. Cambridge Studies in Magnetism. Eds. D. Edwards, D. Melville. Cambridge: Cambridge University Press, 1993.

[13] P. Fulde. Electron Correlation in Molecules and Solids. Solid State Sciences. V. 100. Third Edition. Berlin: Springer, 1995.

[14] J. Hubbard. Proc. Roy. Soc. A. 1963. V. 27b. P. 233; М. И. Владимир, В. А. Москаленко. TMФ. 1990. T. 82. C. 428.

[15] J. E. Hirsch. Phys. Rev. Lett. 1985. V. 54. P. 1317.

[16] В. А. Москаленко. ФММ. 1959. Т. 8. C. 503.

[17] H. Suhl, B. T. Matthias, L. R. Walker. Phys. Rev. Lett. 1959. V. 3. P. 552.

[18] L. M. Falicov, J. C. Kimball. Phys. Rev. Lett. 1969. V. 22. P. 997.

[19] Н. Н. Боголюбов. ЖЭТФ. 1958. Т. 34. № 1. С. 58; Избранные труды. Киев: Наукова думка, 1972.

Поступила в редакцию 21.XI.2000 г., после доработки 15.I.2001 г. 